\title{
Doença de Dupuytren. Revisão de 100 doentes operados
}

\author{
D. Nuno Alegre, L. Azevedo, N. Ferreira, M. Liça, P. Peixoto, F. Silva, \\ J. Mendes, A. Faria, C. de Sousa
}

SERVIÇO de Ortopedia do Hospital Padre AmÉrico.

VALE DO SOUSA.

Correspondencia:

E-mail: duartealegre@yahoo.com

\begin{abstract}
A Doença de Dupuytren observa-se com frequência nesta região. Dada a sua incidência, a diminuição da capacidade de trabalho que pode provocar e o elevado número de casos tratados cirurgicamente, decidimos reavaliar os pacientes, tentando identificar factores etiológicos e o resultado do tratamento.

Foi efectuado um estudo de revisão clínico e de registos em processo de 100 casos, operados entre Fevereiro de 2002 e Dezembro de 2005 no Serviço de Ortopedia do Hospital do Vale do Sousa.

Após discussão com os ortopedistas assistentes, todos os dados relevantes recolhidos foram tratados estatisticamente em colaboração com o Serviço de Bioestatística da Faculdade de Medicina do Porto.

Os resultados são precedidos de uma perspectiva histórica e um sumário da etiologia, evolução, estadiamento, classificação e opções de tratamento da doença de Dupuytren.
\end{abstract}

Palabras clave: Doença de Dupuytren.
Dupuytren's disease is observed frequently in our region. Given its incidence, the diminution in the capacity to work that it can cause, and the high number of cases treated surgically, it was decided to review this patients, trying to identify etiological factors and the results of treatment.

A review was carried out, clinical and of notes of 100 cases, intervened between February 2002 and December 2005 at the Department of Orthopaedics of the Hospital do Vale do Sousa.

After discussion with the orthopaedic surgeons, all relevant data were collected and the statistical analysis was done in collaboration with the Statistical Service of the Faculdade de Medicina do Porto.

Our results are presented, with an historical perspective, and a summary of aetiology, evolution, stages, classification and treatment options for Dupuytren's disease.

Key words: Dupuytren's disease.

Rev. Iberam. Cir. Mano - Vol. 34 • Núm. 2 - noviembre 2006 (49-55) 


\section{INTRODUÇÃO}

\section{Definição}

Doença de Dupuytren - Fibroplasia proliferativa do tecido subcutâneo palmar, na forma de nódulos e cordões, com contracturas secundárias por espessamento da fáscia palmar e seus prolongamentos digitais.

\section{Anatomia}

O complexo fascial palmar aparece à $5^{\mathrm{a}}$ semana de vida intra-uterina, permite a sustentação da pele às estruturas profundas além de estabilizar os metacarpianos.

Esquematicamente:

- Fáscia Palmar:

Central: Fibras longitudinais

Fibras transversais

Fibras verticais

Tenar: Aponevrose tenar

Hipotenar: Aponevrose hipotenar

- Palmo-digital: Bandas espirais de Gosset Ligamento natatório

- Digital: Ligamentos de sustentação do aparelho extensor, cutâneos, fáscia digital lateral e retrovascular de Thomine

\section{Resenha Histórica}

A primeira referência à doença é atribuída a Felicis Plater que a descreveu como uma lesão dos flexores num escultor (in Observatiomini in Hominis, Basileia, 1613)

Em 1808, Henry Cline refuta a lesão dos flexores como etiologia e dá incio à fasciotomia percutânea.

A 1823, Sir Astley Cooper descreve a contractura distinguindo-a das produzidas por lesão dos flexores

Guillaume Dupuytren em 1831, apresenta aos seus alunos um caso de contractura da fascia palmar num cocheiro. Um ano mais tarde, Dupuytren publica um artigo descrevendo de forma minuciosa a patologia e tratamento cirúrgico num comerciante.

No ano de 1900, Keen populariza excisão radical da aponevrose palmar.
Em 1959, Luck demonstra que a lesão básica da doença é uma proliferação fibroblástica que tende a unir pele à fascia palmar.

\section{Epidemiologia}

Sendo mais frequente no sexo masculino (410:1) e com uma idade média de aparecimento de 50 anos, a incidência mundial segue o trajecto migratório das populações vikings, sendo mínima em África e na Ásia e elevada nos países nórdicos (Noruega 10,5\% ○"; 3,1\% \%). Na Austrália, país com forte imigração caucasiana, $20 \%$ dos homens com mais de 60 anos apresentam doença de Dupuytren.

A mão direita é dominante mas a bilateralidade é muito comum. Cerca de $1 / 3$ dos casos da doença atingem um só dedo, 1/3 dois e 1/3 mais de dois, sendo o dedo anelar o mais afectado, seguido do dedo mínimo.

A história familiar é positiva em $30 \%$ dos casos

Está descrita uma associação com doença de Peyronie e Ledderhose e as taxas de recidiva são variáveis conforme as séries estudadas $(0 \%$ $71 \%)$.

\section{Etiologia}

A verdadeira etiologia é desconhecida, havendo algumas teorias descritas:

1) Teoria intrínseca: atribuí a origem à aponevrose palmar pré-existente

Teoria extrínseca: metaplasia do tecido fibroadiposo

3) Teoria mista: defendida por Gosset

4) Teoria histoquímica:

» alterações da composição e distribuição dos ác. gordos<smiles>[AlH2]</smiles>

» alteração da relação colagéneo III / I; $\uparrow$ activ. lisil-oxidase $\gg \uparrow$ radicais livres<smiles>[AlH2]</smiles>

isquemia pele / tec. subcutâneo $\rightarrow$ hipóxia<smiles>[AlH2]</smiles>
proliferação/estimulação fibroblastos $\downarrow$ Dupuytren 


\section{Patologias associadas}

Estão descritas a Diabetes, o Alcoolismo, o Tabagismo, a Doença de Ledderhose $(5 \%)$ e a Doença de Peyronie (3\%). O uso de fármacos para a Epilepsia (fenobarbital) surge associado ao Dupuytren em vários estudos. História de traumatismos parece não estar relacionado na maioria das séries estudadas.

\section{Classificações}

- Classificação de Luck*:

$\gg$ Estadio Proliferativo

» Estadio Involutivo

» Estadio Residual

*classificação mais funcional/fisiológia mas pouco utilizada

\section{- CLASSIFICAÇÃO DE TUBIANA**:}

» Estadio 0 - Sem lesão

» Estadio N - Nódulo palmar ou digital sem flexão

» Estadio I a IV - grau total de flexão somando graus de contractura das diferentes articulações implicadas (Figura 1).

» Polegar - Estadio 0, I, II e III (ângulo I o/II ${ }^{\circ}$ metacarpo) $70^{\circ}-0^{\circ}$

** a classificação mais utilizada no estudo da doença de Dupuytren

- CLASSIFICAÇÃO DE MIKKELSSON***: Tubiana modificada

O Estadio $\mathrm{N}$ da Classificação de Tubiana é o Estádio I da Classificação de Mikkelsson, sendo o Estádio I a IV daquela, o II a V desta.

***por nos parecer mais práctica e de fácil aplicação foi a escolhida neste estudo.

\section{Clínica}

Nódulos palmares $\rightarrow$ Cordas palmares

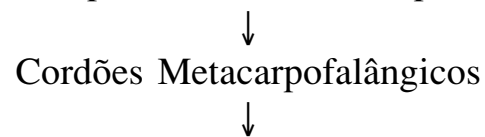

Cordões Interfalângicos Proximais<smiles>[AlH2]</smiles>

Deformidade em flexão dos dedos em grau variável

$\downarrow$

$4^{\circ}$ e $5^{\circ}$ raios / dedos mais frequentemente afectados

(75\% dos casos começam pelo $4^{\circ}$ ou $5^{\circ}$ raio)

A doença é bilateral em $45 \%$ dos casos mas raramente de forma simétrica. A sintomatologia é tipicamente pobre: prurido e dor raramente associados aos nódulos.

\section{Diagnóstico}

Essencialmente clínico, os exames auxiliares de diagnóstico são, por definição, desnecessários.

\section{Tratamento}

- Tratamento conservador ?!

- Indicação cirúrgica clássica:

$30^{\circ}$ de contractura da MCF e qualquer grau de flexão da IFP

- Tipos de cirurgia*:

1. Fasciotomia percutânea.

2. Fasciectomia parcial ou selectiva.

3. Fasciectomia total ou completa.

4. Fasciectomia com enxerto de pele.

5. Amputação.

* Associadas a capsulotomia articular em estadios avançados.

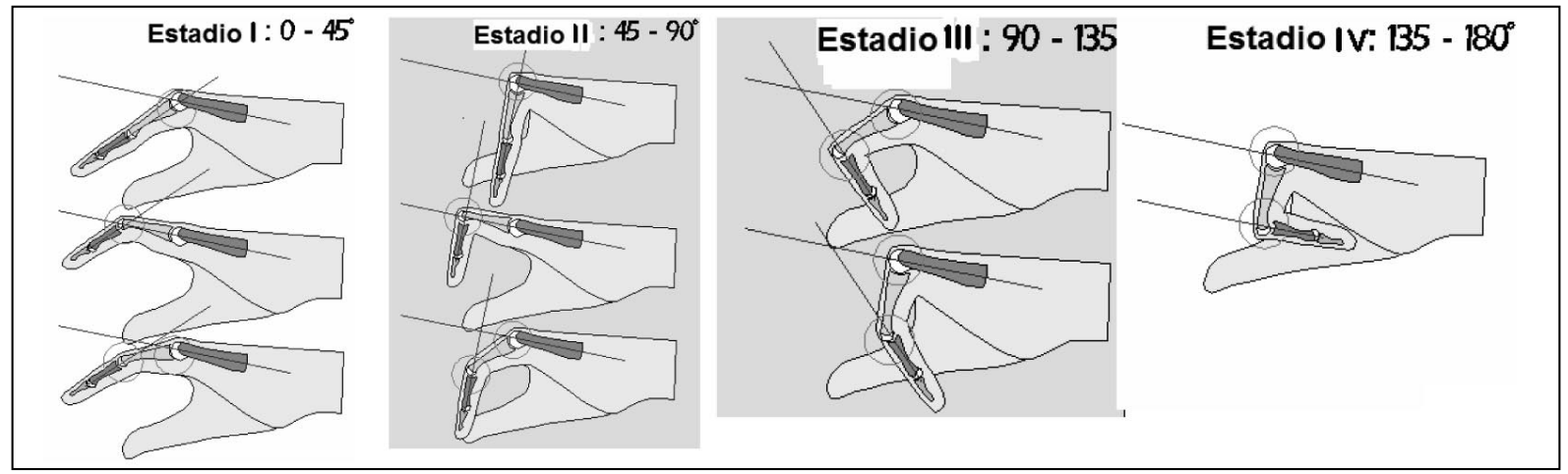

Figura 1. 


\section{Prognóstico}

- Evolução da doença com padrões variáveis.

- Deformidade notória requer vários anos...

- Regressão é rara.

- Recidiva pós-cirurgia dependente de diversos factores.

- Factores de pior prognóstico:.

1. História familiar positiva.

2. $\sigma^{\prime}$ : mais agressivo $q:>$ índice de recidivas.

3. Alcoolismo.

4. Epilepsia.

5. Bilateralidade.

6. Associação a fibromatose plantar.

7. Evolução prévia da doença agressiva.

\section{MATERIAL E MÉTODOS}

De um total 103 doentes operados de Fevereiro de 2002 a Dezembro de 2005, tivemos acesso a 100 processos clínicos, tendo realizado 65 entrevistas com anamnese e exame objectivo segundo protocolo de avaliação realizado em colaboração com o Serviço de Bioestatística da Faculdade de Medicina da Universidade do Porto.

Análise da amostra global de doentes com Doença de Dupuytren (Amostra de 100 indivíduos)

\begin{tabular}{|c|c|c|c|}
\hline & & Count & Column $\%$ \\
\hline \multirow[t]{3}{*}{ Respondeu? } & Năo respondeu & 35 & $(35,0 \%)$ \\
\hline & Respondeu & 65 & $(65,0 \%)$ \\
\hline & Total & 100 & $(100,0 \%)$ \\
\hline \multirow[t]{3}{*}{ Sexo } & $\mathrm{F}$ & 24 & $(24,0 \%)$ \\
\hline & M & 76 & $(76,0 \%)$ \\
\hline & Total & 100 & $(100,0 \%)$ \\
\hline \multirow[t]{6}{*}{ Anestesia } & local & 11 & $(11,0 \%)$ \\
\hline & plexo & 20 & $(20,0 \%)$ \\
\hline & geral & 69 & $(69,0 \%)$ \\
\hline & NA & 0 & $(, 0 \%)$ \\
\hline & NR. & 0 & $(.0 \%)$ \\
\hline & Total & 100 & $(100,0 \%)$ \\
\hline \multirow[t]{4}{*}{ Tipo de cirurgia } & $\begin{array}{l}\text { Fasciectomia } \\
\text { palmar selectiva }\end{array}$ & 46 & $(46,0 \%)$ \\
\hline & $\begin{array}{l}\text { Fasciectomia } \\
\text { palmar digital } \\
\text { selectiva }\end{array}$ & 52 & $(52,0 \%)$ \\
\hline & Amputag:ao & 1 & $(2,0 \%)$ \\
\hline & Total & 100 & $(100,0 \%)$ \\
\hline \multirow{4}{*}{$\begin{array}{l}\text { Lateralidade das } \\
\text { legdes }\end{array}$} & B & 42 & $(42,0 \%)$ \\
\hline & D & 33 & $(33,0 \%)$ \\
\hline & $E$ & 25 & $(25,0 \%)$ \\
\hline & Total & 100 & $(100,0 \%)$ \\
\hline
\end{tabular}

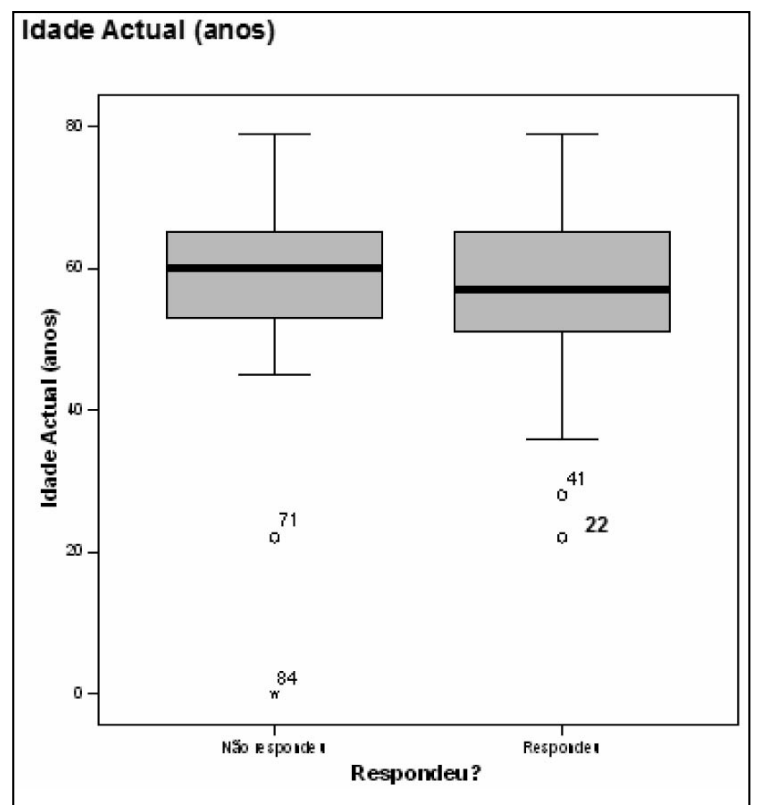

Figura 2.

A nossa amostra é constituída por doentes com uma média de idades de 58 anos (Figura 2) e na sua maioria do sexo masculino $(79 \%)$. A história familiar revelou-se positiva em $26 \%$.

Em $42 \%$ dos casos, os doentes apresentavam doença bilateral e em $33 \%$ apenas à direita (mão dominante).

O $4^{\circ}$ raio foi o mais afectado (77\%), seguido do $5^{\circ}(58 \%)$ e $3^{\circ}$ raio (26\%). A maior parte dos doentes apresentaram $1(45 \%)$ ou $2(48 \%)$ raios atingidos.

Os primeiros sinais referidos pelos doentes foram nódulos palmares (Figura 3).

De salientar a elevada prevalência da diabetes, dislipidemia, hiperuricemia e patologia hepática como doenças associadas (Figura 4).

Doenças como Peyronie e Ledderhose, apresentaram prevalências superiores à população em geral (Figura 4).

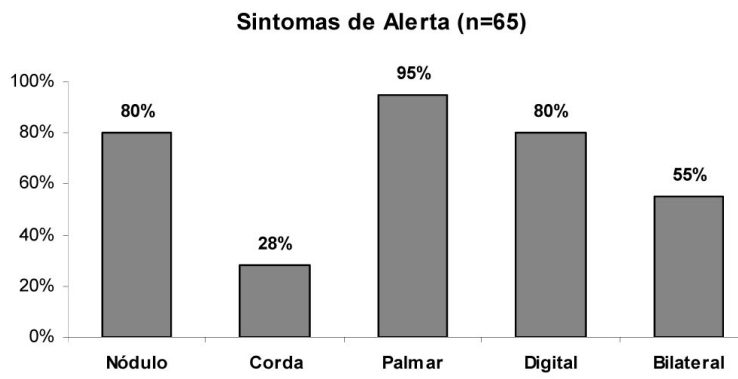

Figura 3.

Revista Iberoamericana de Cirugía de la Mano 


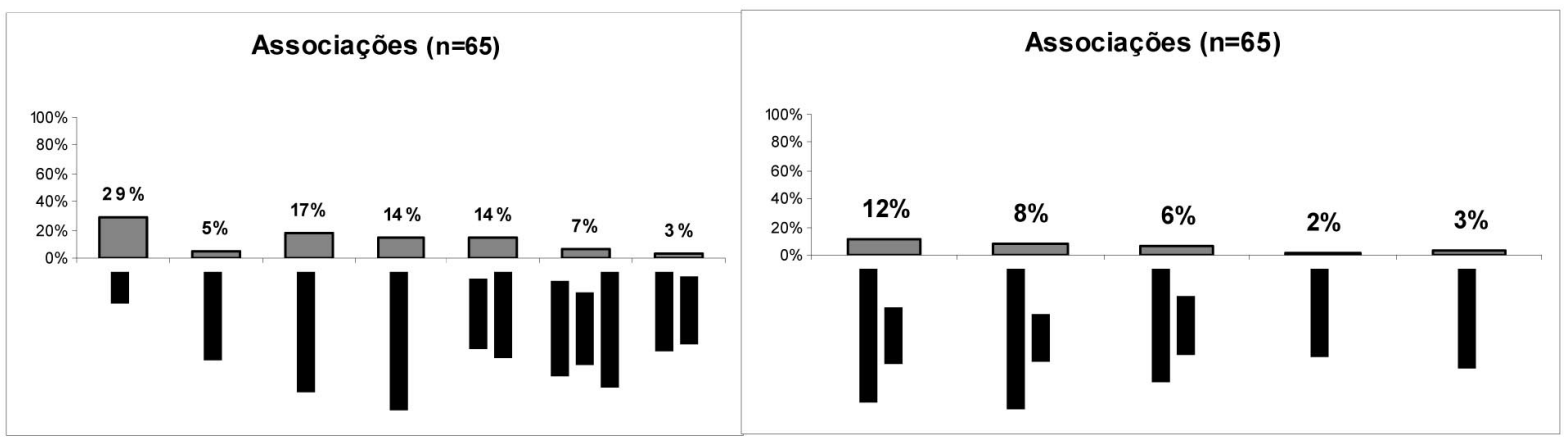

Figura 4.

Apenas $23 \%$ dos doentes da amostra negaram ingestão de álcool, estando os hábitos tabágicos presentes em $42 \%$ dos casos.

\section{RESULTADOS}

Neste grupo, a maioria dos doentes apresentavam Mikklesson II, III e IV (Figura 5), tendo sido submetidos a fasciectomias selectivas sob anestesia geral. $80 \%$ dos doentes foram submetidos a apenas uma cirurgia (Figura 6).

$\mathrm{Na}$ maioria dos doentes não houve complicações no pós-operatório, no entanto, destacamos as alterações de sensibilidade que nos casos em que não houve desaparecimento total, constatou-se que a sintomatologia tendeu a esbater-se com o tempo e a necrose da pele presente nos cuidados de penso pós-operatório em $11 \%$ dos casos (Figura 7).

Verificaram-se recidivas em $14 \%$ dos doentes operados.

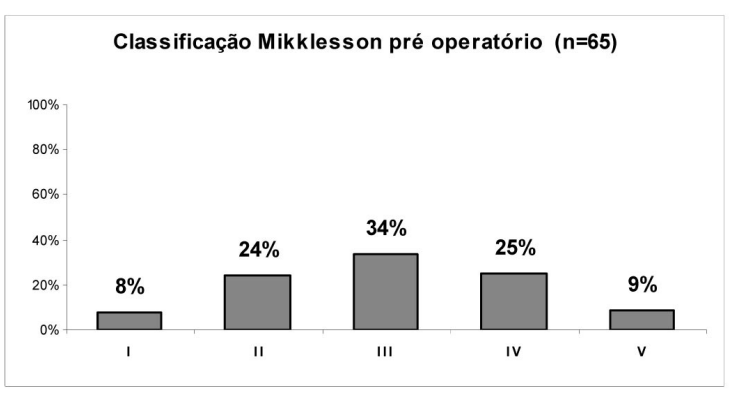

Figura 5

\section{DISCUSSÃO}

- A amostra deste estudo não difere da classicamente descrita nesta doença.

- Associações mais importantes:

- Alcoolismo (Figura 8).

- Diabetes Mellitus.

- Antecedentes familiares de doença de Dupuytren

- Os doentes operados distribuiram-se entre o Grau I e V de Mikkleson pré-operatório (Figura 14).

- Apenas um caso foi submetido a amputação

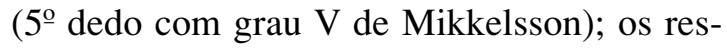
tantes a fasciectomia selectiva

- O défice de mobilidade e a presença de cicatriz dolorosa revelaram-se como as sequelas mais frequentes (Figura 9).

O grau de satisfação dos doentes foi em $77 \%$ dos casos satisfeito $(60 \%)$ ou muito satisfeito $(17 \%)$ e em $23 \%$ dos casos pouco (18\%) ou nada satisfeito (5\%) (Figura 16):

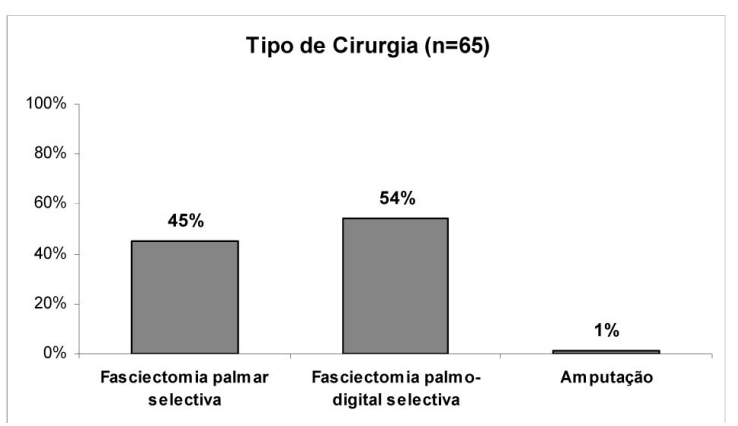

Figura 6 


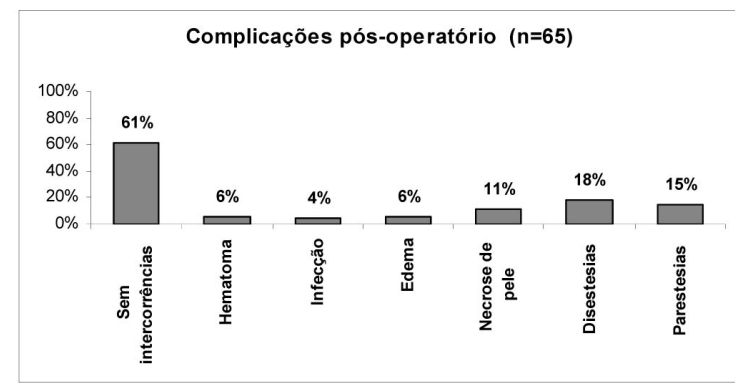

Figura 7.

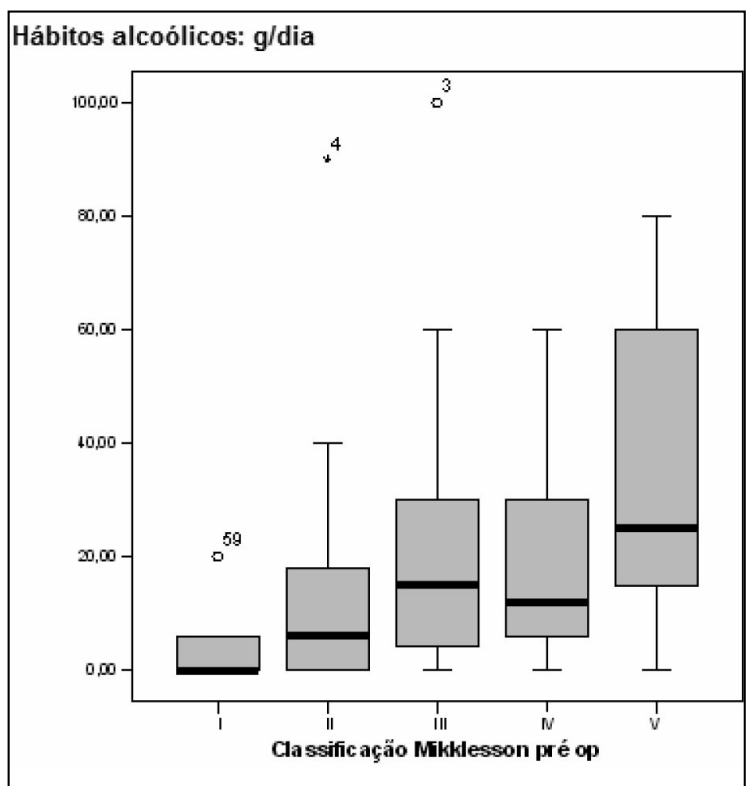

Figura

A maioria dos doentes operados $(82 \%)$ recomendava a cirurgia a um amigo com Doença de Dupuytren

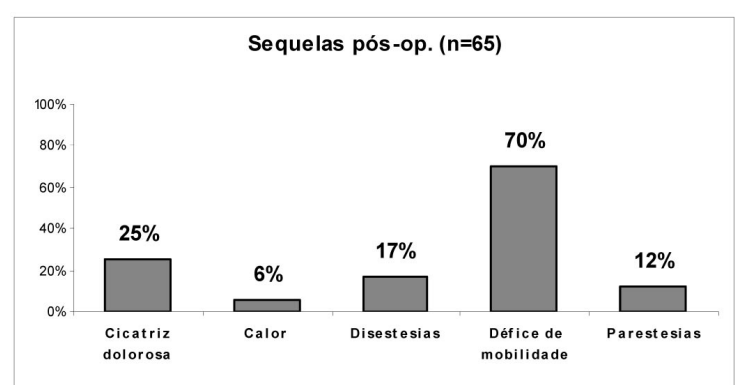

Figura 9.

$\mathrm{O}$ facto de termos uma amostra recente terá contríbuido para o baixo índice de recidivas apresentado

\section{CONCLUSÕES}

A doença de Dupuytren constitui uma doença evolutiva da mão, uma das zonas mais nobres do corpo humano, cuja progressão incapacitante pode ser interrompida de forma eficaz com o recurso a tratamento cirúrgico.

No nosso grupo de estudo, embora com valores estatisticamente não significativos, encontrou-se uma relação directa entre grau de satisfação e :

- Menor gravidade pré-operatória

- Uso de tala (25\%) e fisioterapia (18\%) no pósoperatório

- Ausência de história familiar

- Ausência de hábitos alcoólicos

\section{BIBLIOGRAFÍA}

1. Doença de Dupuytren. Análise de 125 doentes (Rev. Port. Ort. Traum., 7: 299-303, 1999) Autores: Marisa Mendes, A. Silva, C. Pinho, A. Martins, J. Reis, J. Amarante

2. McFarlane RM. The current status of Dupuytren's disease. $\mathrm{J}$ Hand Ther 8:181, 1995.

3. Robert M.McFarlane. «Enfermedad de Dupuytren». En Cirugía Plástica. La mano. Tomo II: Editor: McCarthy, May,Littler. Editorial Médica Panamericana S.A.- Madrid - España, 1992. Pp.819-851.
4. Rober M.McFarlane. «Dupuytren's Contracture». En Operative Hand Surgery.Third Edition. Volume 1: Editor: David P. Green,MD. Churchill Livingstone. New York, 1993. Pp 563 - 591

5. Hand Clinics. « Dupuytren's Disease». Volume 15. Number 1.Guest Editor: Ghazi M. Rayan. W.B. Saunders Company, Philadelphia. February 1999.

6. James D. Saar, M.D., and Peter C.Grothaus, M.D. « Dupuytren's disease: An Overview «Plast Reconstr Surg» 106: 125-134, 2000
7. Dupuytren G.: De la retraction des doigts par suite d'une affection de Papponeurose palmaire. J. Univ. Med. Chir. Paris. $5-352 ; 1831$.

8. Meinel A. Description of Dupuytren disease by the Basel physician and anatomist Félix Plater in 1614 (letter; coment). Handchir Mikrochir Plast Chir 1.995 Sep; 27(5): 272-5 3- Badalamente. MAStren. L., and Hurst

9. L.C. The pathogenesis of Dupuytren's contracture; contractile mechanisms of the myofibro- 
blasts. J. Hand Surg. 8-235, 1983.

10. Bayley, A.J. Sims, T.J., Gabbiani, G., Bazin.S. and L-Lous, M. Collagen of Dupuytren's disease. Clin.Sci.Mol. Med., 53: 499, 1977.

11. Bennett B.: Dupuytren's contracture in manual workers. Br. J. Indust. Med. 39:98, 1982.

12- Crisp, A.J., and Heathcote, J.G: conective tissue abnormalities in diabetes mellitus. J. R Coll. Physicians Lond.,18: 132, 1984.

13- Critchley,E.M.R, Vakil, S.D., Hayward,H.W. and Owen, V.M.H.: Dupuytren's disease in epilepsy: reslt of prolonged administration of anficonvulsant. J. Neurol. Neurosurg. Psychiatry, 39: 498, 1976.

14. Su, C. K:and Pattek, A.J., Jr.: Dupuytren's contracture. Its association with alcoholism and cirrhosis. Arch.Imern Med., 126: 278, 1970.

15. Watson,J.D.: Fasciotomy and Z-plasty in the management of Dupuytren's contracture. Br.J. Plast.Surg.,37:27, 1984.

16. Meinel A.: Dupuytren disease formal pathogenesis without contraction and a new surgery concep. Langenbecks Arch Chir Suppl Kongressbd. 1998: 115:1292-4.

17. Dohlem.R On permanent retraction of the fingers, according to Dupuytren. Rev. Rhum. Engl. Ed. 1996 Jun; 63(6): 435-43.
18. Mikkelsen O A (1990) Epidemiology of a Norwegian population. In: McFarlane R M (ed) Dupuytren's Disease. Churchill Livingstone, Edinburgh, pp 191200 Moermans J P, Duchateau J (1984) 19. La maladie de Dupuytren: résultats d'une technique simplifiée. Revue Médicale de Bruxelles 5:467-471 20. Gelbard, M.K., Lindner, A., Kaufman, J.J. The Use of Collagenase in the Treatment of Peyronie's Disease. J. of Urology: 134, August 1985, 280-283

21. Gelbard, M.K., Walsh, R., Kaufman, J.J. Collagenase for Peyronie's Disease Experimental Studies. Urol. Res. (1982) 10:135-140

22. Hamilton, R.G., Mintz, G.R., Gelbard, M.K. Humoral Immune Responses in Peyronie's Disease Patients Receiving Clostridial Collagenase Therapy. J. of Urology 135: 641-647, March 1986

23. Gelbard, M.K., James, K., Riach, P., Dorey, F. Collagenase Versus Placebo in the Treatment of Peyronie's Disease: A Double-Blind Study. J. of Urology Vol. 149: 56-58 January 1993

24. Majno G, Gabbiani G, Hirschel BJ, et al. Contraction of granulation tissue in vitro. Science 173: 548, 1971.

25. McFarlane RM. Unpublished data from the Dupuytren's di- sease committee of the International Federation of Societies for Surgery of the Hand, 1985.

26. Bower M, Nelson M, Gazzard BG. Dupuytren's contracture in patients affected with HIV. BMJ 300:164, 1990.

27. Ling RSM. The genetic factors in Dupuytren's disease. J Bone Joint Surg 45B:709, 1963.

28. Matthews P. Familial Dupuytren's contracture with predominantly female expression. $\mathrm{Br} \mathrm{J}$ Plast Surg 32:120, 1979.

29. McFarlane RM. Dupuytren's disease: relation to work and injury. J Hand Surg (Am) 16:775, 1991.

30. Liss GM, Stock SR. Can Dupuytren's contracture be work related? Review of the evidence. Am J Indus Med 29:521, 1996.

31.Classen DJ, Hurst LN. Plantar fibromatosis: a case report of bilateral flexion contractures and review of the literature. Ann PI Surg 28:475, 1992.

32. Hueston JT. State of the art: the management of recurrent Dupuytren's disease. Eur Med Bibliography 1: 7,1991.

33. Marques $M$, Silva $A$, Cordeiro MNDS; Pinho C, Martins A, Amarante $\mathrm{J}$ Enfermedad de Dupuytren: particularidades de la población Portuguesa. Cir Plást Iberlatinamer 2002; 28: 125-129). 